\title{
Ri-leggere il welfare state in una prospettiva di genere
}

\section{GÉNERO Y EDUCACIÓN}

\section{Por: Stefania Ruggeri}

Univerità del Salento - Dipartimento di Storia

Società e Studi sull'Uomo - Lecce - Italia

e-mail: stefania.ruggeri@unisalento.it

\begin{abstract}
Il saggio analizza alcune delle questioni cruciali connesse con il tema della "cura", emerse all'interno dei più ampi processi di cambiamento generati dal progressivo invecchiamento della popolazione e dalla crisi della famiglia basata sul modello male beadwinner, cambiamenti che fanno del "care" la 'nuova' questione sociale. Utilizzando un paradigma di genere, il lavoro affronta il tema dell'invecchiamento, tema di grande attualità non solo per le difficoltà connesse con la sua sostenibilità sociale ma anche per le impreviste e inattese ripercussioni che esso ha sulla famiglia in termini di distribuzione delle responsabilità nel lavoro di cura.
\end{abstract}

\section{Parole chiave}

Genere, cura, welfare mediterraneo, invecchiamento

\section{II ruolo della famiglia nel welfare mediterraneo}

Negli anni novanta, l'analisi del ruolo della famiglia nella triade famiglia-statomercato, già sottolineata nel proficuo dibattito avviato dalla teoria sul welfare di Esping Andersen (1990), genera nuovi modelli teorici che danno vita ad una riclassificazione gender sensitive dei modelli di Stato sociale e contribuiscono a mettere in evidenza la centralità della famiglia nella stessa formazione dei regimi di welfare (Moreno, 2002).

Nello stesso periodo gli studi di genere (Lewis, 1998; Sainsbury, 1994-1996; O'Connor, 1993; Orloff, 1993) hanno posto l'accento sulla reciproca interazione tra genere e stato sociale dimostrando come differenti sistemi di welfare abbiano favorito il proliferare di diversi tipi di servizi finalizzati ad 
incentivate la presenza della donna nel mercato del lavoro anche attraverso la conciliazione tra vita professionale e responsabilità familiari (Leira, 1992).

Questi studi hanno messo in evidenza non solo la questione del caring, facendo emergere il lavoro non remunerato e l'accesso alla cittadinanza come una questione di genere, ma anche il ruolo dei caregivers, indicando nella esternalizzazione di questi servizi una delle alternative possibili per una riassegnazione dei compiti di cura (commodification of care) (Hochschild, 2000 a). Facendo riferimento ad una concezione più ampia della cittadinanza sociale, questi lavori hanno contribuito a mettere in luce, infine, come il diritto a vedere soddisfatti i propri bisogni di cura e il dovere nel prestare cura siano una questione centrale non solo per le donne ma per ogni cittadino (Kittay, 1999). Una questione non più rinviabile alla luce dell'attuale care deficit e in considerazione dei mutamenti demografici in atto nelle società occidentali.

A partire da questi studi sono nati nuovi approcci che hanno sottolineato il ruolo delle migrazioni nella ricerca di una soluzione possibile ai problemi di assistenza, attraverso la formula love and gold for woman nel lavoro di cura (Hochschild, 2000 a), facendo emergere nel "welfare delle connessioni" il filo diretto tra la gendered nature of transnational movemnets e welfare state (Piperno, Tognetti Bordogna, 2012).

In questo modo, dunque, la questione della famiglia entra come concetto analitico nella differenziazione dei modelli diwelfare, svolgendo lo stesso ruolo che il concetto di 'regime di welfare' aveva avuto nello studiare somiglianze e differenze tra i diversi sistemi nazionali (Flaquer, 2000). Essa diviene una delle caratterizzazioni dei vari modelli di welfare poiché la sua centralità risiede nella ripartizione delle responsabilità con lo Stato e il mercato (Martin, 1996).

Queste considerazioni hanno consentito, ampliando l'articolazione proposta da Esping Andersen (1990-1999), I'individuaziane di un quarto modello caratterizzato da una logica di sviluppo diversa rispetto a quella dei regimi occidentali. Si tratta del Modello Mediterraneo che caratterizza i sistemi di welfare di Italia, Spagna, Grecia e Portogallo (Ferrera, 1993 a, Moreno e Sarasa, 1992, Moreno, 2002). Paesi "dimenticati" negli studi tradizionali sul welfare (Esping Andersen, 1990-1999) nei quali Portogallo e Grecia e, in misura 
minore Italia e Spagna, sono presi in considerazione in modo marginale in quanto caratterizzati da regimi definiti conservatori, arretrati e familisti.

La maggior parte delle ricerche classiche sul welfare state, infatti, avevano posto l'acento sugli schemi di sicurezza sociale, sulle forme di copertura assicurativa, sui soggetti aventi diritto, concentrando l'attenzione sulle misure sociali previdenziali (l'assicurazione contro gli infortuni, la pensione, i sussidi di disoccupazione, ecc.), su quelle assistenziali (le misure a sostegno del reddito) e sulla sanità, ritenute rilevanti nella definizione del welfare state. In esse, come afferma Naldini (2002), poco peso era stato dato, invece, allo studio dell'evoluzione delle politiche sociali rivolte al sostegno delle responsabilità familiari, nonché alle norme che definiscono ex lege le obbligazioni familiari e indirizzano in tal senso lo sviluppo delle politiche sociali.

Con la messa al centro della famiglia, in contrapposizione alla tesi che definisce i paesi del litorale latino come semplicemente caratterizzati da un welfare state meno evoluto, il dibattito sul tema si focalizza sull'opportunità di accogliere la tesi (Leibfried, 1992) secondo cui questi paesi hanno in realtà sviluppato un sistema di welfare autonomo, che viene descritto come una sottocategoria all'interno dei regimi continentali (Esping Andersen, 1999), una sorta di via intermedia tra la tradizione bismarckiania e quella beveridgiana (Moreno, 2001). Sono Paesi, segnala Ferrera (1998 a), che condividono tra loro, oltre all'influenza della Chiesa e ad un marcato familiarismo, alcune caratteristiche istituzionali e politiche: la presenza di programmi di trasferimento del reddito caratterizzati o da eccessi di protezione (pensioni) o al contrario da totale scopertura in alcune aree (servizi alle famiglie); una tradizionale prevalenza di trasferimenti monetari alle persone rispetto alla fornitura diretta di servizi sociali; la presenza di un apparato statale alquanto debole e lacunoso e alla mercé di reti di potere di tipo clientelare.

Ferrera (1998 a) e Flaquer (2001) individuano, inoltre, alcuni tratti tipici del welfare mediterraneo nella presenza di sistemi di garanzia del reddito frammentati per categorie occupazionali; in un sistema fondato su un dualismo tra beneficiari "forti" e "deboli"; nel ruolo centrale della famiglia nel compensare le carenze del welfare (Moreno, 2002), mostrando come, laddove le politiche familiari risultano 'sottosviluppate', la famiglia rimanga il principale fornitore di benessere, lasciando al mercato e allo stato un ruolo residuale e controllato (Daly e Lewis, 2000). 
Un altro elemento di affinità tra i Paesi dell'area del Mediterraneo è rappresentato, infine, dalla presenza di un sistema sanitario universalistico che rende l'assistenza sanitaria un diritto per tutti i cittadini (Saraceno, 1998) e da una carente rete di servizi sociali e di assistenza, che lascia alla famiglia la scelta di operare secondo proprie strategie ed iniziative private (Climent, 2008).

Altri autori (Moreno 2002; Naldini, 2006) hanno poi implementato l'elenco delle connotazioni peculiari al welfare mediterraneo, segnalando altri fattori istituzionali comuni a questo regime: l'importanza dell'economia sommersa e l'invisibilità dei mercati "duali" del lavoro (Doeringer, Piore, 1971); I'esistenza di precedenti storici comuni, in particolare per Grecia, Italia, Portogallo e Spagna che hanno sperimentato dittature fasciste e che hanno vissuto i processi di industrializzazione e modernizzazione in tempi del tutto diversi rispetto ad altri Paesi europei (Moreno, 2004).

Tra le caratteristiche comuni ai quattro Paesi citati (Casles, 1995; Leibfried, 1992; van Kersbergen, 1995) un posto di rilievo ha il ruolo che la Chiesa cattolica ha avuto nel radicare culturalmente principi di solidarietà e sussidiarietà (parte integrante del familismo) che hanno da sempre contribuito a legittimare l'intervento pubblico solo nella inadeguatezza delle reti primarie (Sainsbury, 1999). Principio di sussidiarietà che ancora oggi si fonda sul modello di famiglia male breadwinner (Sainsbury, 1999) al quale lo Stato delega le responsabilità della cura e della sussistenza astenendosi dallo sviluppo di politiche family friendly (Esping Andersen, 1996).

Un emblematico esempio di solidarietà familiari e intergenerazionali è rappresentato dal caso spagnolo delle supermujeres (Moreno, 2002): mogli, madri, nonne che, all'interno della famiglia estesa partecipano, ad una gara di resistenza, un tour de force ai limiti delle umane capacità, sostituendosi quasi interamente allo Stato. Questo contributo, per così dire "eccessivo", della famiglia alla protezione sociale, fa sì che alcuni autori sostengano che il deficit del sistema di welfare mediterraneo non sia causato dalla sua bassa de-mercificazione ma dalla bassa defamiliarizzazione (Flaquer, 2001). 
In uno studio comparato del caso Italiano e Spagnolo, Naldini (2002) evidenzia come la famiglia mediterranea, si caratterizzi in modo autonomo e specifico pur all'interno del modello classico del male breadwinner, la cui piena realizzazione è stata ostacolata dalla concomitanza di molti fattori: la presenza di una struttura occupazionale di tipo agricolo; l'esistenza di aree lavorative sommerse; alti livelli di emigrazione segnati da una mancata occupazione maschili. Sia in Italia che in Spagna, continua Naldini, il regime di welfare, più che sul modello forte del capofamiglia, si fonda sulla percezione della famiglia come unità di reddito e risorse, alla quale tutti i membri contribuiscono secondo le proprie possibilità, un sistema fondato sulla solidarietà familiare che distribuisce la responsabilità primaria nelle prestazioni di cura, alle donne sposate e madri all'interno di una rete familiare allargata femminilizzata (Saraceno, 1994).

Nel regime mediterraneo, dunque, le performance della famiglia si configurano come un elemento di 'diversità' (Moreno, 2004) che, afferma Flaquer (2000), risiede nella presenza di una circolarità tra la mancanza di misure tese all'implementazione di politiche familiari e l'assenza di domanda di tali misure. Questo paradosso sembra essere una sorta di profezia che si autoadempie, che crea un feedback negativo in una situazione in cui non solo il sistema si riproduce ma si rafforza (Flaquer, 2000). Le donne sposate adulte, motivate da un senso di obbligo morale, diventano le principali sostenitrici di questo paradosso che si nutre di abnegazione, interazioni giornaliere, vicinanza, coabitazione tra i membri della famiglia.

\section{Il lavoro di cura tra vecchie certezze e nuove definizioni}

Il dibattito accademico, innescato dal movimento femminista, apre la strada ad una accurata riflessione teorica nel campo delle scienze sociali intorno al concetto di cura (Wærness, 1996): I'interesse delle femministe, rivolto agli aspetti quotidiani e concreti del care, fa emergere la vasta gamma di contenuti in esso impliciti (Daly e Lewis, 2000) contribuendo a delinearne i tratti caratteristici fondati sul suo essere inevitabilmente legato alla sfera affettiva (Kittay, 1999). Attorno a questi argomenti viene ridefinito lo stesso concetto di lavoro, che assume connotazioni diverse a seconda del campo di osservazione: lavoro riproduttivo, domestico-familiare o lavoro non remunerato (Waerness, 1987). 
In questi anni, infatti, il lavoro assume una parte rilevante nelle analisi di genere divenendo un esteso campo di ricerca, in quanto dimensione materiale e simbolica in grado di restituire alle donne visibilità e cittadinanza. A partire dalla decostruzione del lavoro per amore, si rivendica in sostanza l'accezione di lavoro alle attività domestiche, sottraendole alla loro presunta naturalezza e conferendo ad esse dignità sociale. Tali argomentazioni hanno fatto emergere la necessità di distinguere progressivamente le logiche che caratterizzano il lavoro di cura erogato in famiglia da quelle presenti nel mercato e nello Stato, rendendo il lavoro 'il metro e la misura' attraverso cui le donne contrattano e definiscono la propria identità sociale (Hernes, 1987).

L'ampiezza dei contenuti e delle evocazioni prodotte, tuttavia, fa del care un concetto complesso, articolato ma sfuggente, afferma Paperman (2004), nel quale si intrecciano dimensioni che è difficile analizzare separatamente: se la versione materiale del care si può in qualche modo quantificare, essa non può, allo stesso tempo, essere facilmente separata dal suo corrispettivo affettivo e morale (Kittay, 1999). In effetti proprio quest'aspetto ha reso storicamente invisibile il concetto di care in letteratura, tradizionalmente descritto come un sapere femminile tipico del mondo delle madri, delle figlie, delle sorelle e delle domestiche (Saraceno, 2009), un'insieme di conoscenze e competenze legate e vincolate a responsabilità morali, tramandate da una generazione all'altra, negoziate tra generazioni di donne (Finch e Mason,1993).

Dalle numerose ricerche emerge una forte ambivalenza all'interno delle dimensioni del care nel mentre si evidenzia la simultanea presenza di tendenze opposte, talvolta conflittuali, che vanno da un altruismo obbligato o di una generosità indotta (Land e Rose, 1985) e, nello stesso tempo, si delineano i diritti e le responsabilità dei caregiver e dei beneficiari, le dimensioni cruciali della relazione tra chi riceve e chi dà aiuto, l'ambito sociale e il contesto istituzionale in cui la cura viene esercitata, sia a livello politico che a livello simbolico (Thomas, 1993).

Anche al di fuori delle sue rappresentazioni più tradizionali (Ciarini, 2011), tuttavia, la relazione tra genere femminile e cura continua a vivere fuori e dentro il mito della consueta 'naturalezza' con cui è stata raccontata, 
riproducendo una sorta di continuità nelle aspettative e nelle attese, una fideiussione garantita dai rapporti di sangue (Leira, 1992) e sancita da norme di reciprocità (Gouldner, 1960). In altre parole, possiamo affermare che l'aiuto 'in/condizionato e costante' fornito in contesti familiari rappresenta la più chiara espressione di ciò che si intende per solidarietà intergenerazionale e può essere considerato un buon esempio per comprendere come the power of reciprocity (Diekmann, 2004), il potere delle reciprocità non sia soltanto una prerogativa dei contesti in cui le obbligazioni familiari sono regolamentate de iure, ma costituisca una realtà de facto anche nei sistemi di welfare avanzati (Finch Mason, 1993). Soffermarsi sulla solidarietà intergenerazionale attraverso il tempo dedicato alla cura materiale ed emotiva è, pertanto, il primo passo per conoscere e comprendere la forza dei legami familiari tra le generazioni (Aboderin, 2005).

Da qualunque punto di osservazione la si guardi, dunque, la moneta ha sempre una doppia faccia: reso con amore o per denaro, in cambio di qualsiasi altro tipo di bene materiale o simbolico, il contesto del care rimane lo sfondo di una relazione tra chi riceve e chi dà cura, una questione interindividuale che nasce nella relazione e in essa si estrinseca; un lavoro, secondo le norme sociali generalmente condivise, prestato in situazioni di dipendenza (Wærness, 1996), un lavoro non solo necessario ma vitale per il successo di una società che vuole garantirsi una adeguata sopravvivenza (Kittay, 1999:50).

\section{Il lavoro di cura tra de-familiarizzazione e ri-familiarizzazione}

L'utilizzo del concetto di "cura” nei dibattiti pubblici è andato, dunque, di pari passo con la concettualizzazione della "parità di genere", in particolare rispetto con la redistribuzione delle responsabilità tra donne e uomini. Si tratta di un problema politicamente controverso che da un lato tocca i tradizionali confini tra pubblico e privato e, intervenendo negli affari privati della famiglia, ne profana la sacralità e dall'altro avvicina il piccolo mondo della cura al grande mondo delle burocrazie pubbliche, fondato su un'etica della giustizia propria dello Stato (Siim, 2000).

Attraverso la crescente femminilizzazione della forza lavoro, attraverso la transizione dal lavoro non retribuito a quello retribuito, che ha contribuito allo smantellamento della famiglia male breadwinner (Lewis, 2002), il concetto di cura da un ambito residuale d'attenzione e d'intervento si è affermato come base per il riconoscimento e l'acquisizione di diritti; identificato e codificato nei contesti istituzionali come uno dei risvolti centrali, se pur problematici, del mutamento sociale (Wærness, 1984; Hernes, 1987; Paperman, 2005; Leira e 
Saraceno, 2002). Il percorso teorico rappresenta, dunque, il costante tentativo di rivelare come la separazione tra pubblico e privato, tra produzione e riproduzione sia, di fatto, una costruzione sociale e di potere che divide l'attività umana in sfere gerarchizzate e connotate sessualmente, nel tentativo di restituire vigore e senso ad entrambe.

In questo senso è stato fondamentale distinguere il lavoro di cura da altri tipi di lavoro, sottolineandone l'articolazione in servicing e caring, dimensioni che ne specificano meglio gli attributi e le condizioni del lavoro (Wærness,1987; Kittay, 1999). Secondo Wærness(1987), occuparsi di un marito autosufficiente contribuendo alla realizzazione del suo benessere è servicing, mentre prendersi cura di un bambino, di un anziano non autosufficiente o di un disabile, con le stesse finalità, è caring. Da questo punto di vista l'autonomia e l'indipendenza del caregiver, in particolare delle donne, assume forme diverse a seconda che la relazione tra chi fornisce e chi riceve cura sia simmetrica o di subordinazione. La distinzione concettuale tra servicing e caring assume una certa rilevanza in quanto solo il secondo acquista terreno nei discorsi e nei dibattiti istituzionali come questione meritevole di interesse a livello politico e sociale poiché solo la cura per le persone molto dipendenti o non autosufficienti definisce analiticamente i confini tra Stato e famiglia, tra Stato/famiglia/mercato, segmentando le responsabilità, le prestazioni e i costi della cura (Leira e Saraceno 2002). Emerge in questo modo una sproporzione nel modo in cui vengono percepiti e riconosciuti $i$ bisogni di cura delle persone molto dipendenti (bambini molto piccoli e anziani non autosufficienti) e il lavoro di chi, pagato o non pagato, risponde a questi bisogni (Saraceno, 2009).

Negli ultimi anni, a livello europeo, sono state evidenziate tendenze contrastanti e per alcuni versi contraddittorie che hanno rimarcato linee di conflitto e di confine tra figure in/compatibili. Per un verso le questioni legate alla cura dei bambini sono diventate parte dell'agenda politica e le responsabilità di cura sono state allargate e riconosciute anche ai padri lavoratori, mostrando l'approssimarsi di soluzioni vicine ad una defamiliarizzazione delle donne e una rifamiliarizzazione degli uomini (Saraceno, 2009): tutto questo al riparo di politiche che sostengono le responsabilità e salvaguardano i diritti comunque legati al mondo del lavoro (Leira, 2002). Per altri versi la partecipazioni delle madri al mercato del lavoro è divenuta un agente moltiplicatore di impegni e responsabilità nel momento in cui si è fatta avanti una nuova esperienza della cura (Saraceno, Naldini, 2011). Ancora oggi, nonostante 
I'invecchiamento della popolazione rappresenti una delle sfide più importanti per le società europee contemporanee e future (Brandt, 2011), i discorsi e le politiche di conciliazione tendono a concentrarsi per lo più sulle prime fasi di formazione della famiglia, sottovalutando le esigenze e le problematiche emergenti nelle fasi successive che attualmente rappresentano la forma più diffusa di dipendenza (Saraceno 2010).

Ciò dipende, secondo Saraceno e Naldini (2011), dal fatto che l'aumento della domanda di cura derivante dall'invecchiamento della popolazione e delle parentele non ha avuto un impatto determinante sulla partecipazione delle donne al mercato del lavoro, nel mentre la responsabilità di cura nei confronti di una persona non autosufficiente riguarda una porzione molto più ridotta di persone rispetto a quelle interessate alla cura di bambini piccoli. I risultati della seconda indagine sulla qualità della vita in Europa (2009) evidenziano, infatti, come più della metà delle donne tra i 35 e 49 anni sia impegnata quotidianamente nell'accudimento ed educazione dei figli, mentre l'accudimento di un anziano non autosufficiente riguardi poco più del 15\% delle donne nella fascia di età compresa tra 50 e 64 anni, con un impegno solo in alcuni giorni a settimana. Queste differenze sono imputabili non solo ad una diversa incidenza della popolazione anziana o con qualche disabilità quanto piuttosto alle diverse modalità di percepire le obbligazioni familiari in caso di dipendenza in età anziana e all'esistenza o meno di servizi di cura, nonché alla loro accessibilità e qualità. Le esigenze di cura relative alla non autosufficienza degli anziani, infine, si differenziano notevolmente da quelle dei bambini sia in termini di prevedibilità che di durata. L'accudimento di una persona anziana implica un'assistenza time-intensive non solo sul piano materiale ma anche sul quello morale, in quanto meno 'gratificante' rispetto alla presenza di un bambino (Saraceno, 2010).

\section{Genere e responsabilità di cura}

Il lavoro remunerato e la vita domestica si sono costruite e mantenute nell'immaginario collettivo della nostra società come aree completamente separate di esperienza e di valori perché tradizionalmente assunte come dimensioni di responsabilità in capo a soggetti differenti che si sono dedicati separatamente all'una e all'altra (Campillo Poza, 2010). Un'attribuzione di responsabilità, come afferma Martín Palomo (2008), risolta in una logica binaria, che struttura i compiti, le responsabilità ed i ruoli in base al genere e arriva alle 
consuete distinzioni tra sfera privata e sfera pubblica, partendo dagli ambiti privati e intimi della vita (Habermas, 1998).

Se osserviamo le pratiche sociali, le narrazioni connesse con le strutture di genere all'interno di ogni regime di welfare, ci accorgiamo subito delle differenze che definiscono libertà e scelte individuali non solo nelle gerarchie sociali, connotate sessualmente, ma anche negli accordi taciti tra donne (Binbi, 2009). La divisione del lavoro remunerato da quello non remunerato in base al genere è stato il grande meccanismo di conciliazione delle società industriali democratiche tanto al livello micro, degli individui e delle famiglie, quanto a livello macro (Saraceno e Naldini, 2011) e i problemi con i quali si confrontano e si scontrano uomini e donne non sono altro che il risultato della messa in discussione di questo meccanismo fondato su una stretta divisione sessuale del lavoro, degli spazi e dei tempi (Campillo Poza, 2010).

Negli studi di genere, infatti, il lavoro, sia come attività retribuita che non retribuita, è stato considerato (Leira 1992) la metafora che ha saputo rappresentare meglio le differenze di genere e la "doppia presenza" rimane concettualmente il luogo caratteristico dell'identità sociale e dell'esperienza femminile nelle società contemporanee: esperienza nella quale si cerca di tenere insieme due universi distinti e contrapposti, terreno di contesa e negoziazione del prestigio, dell'identità e del riconoscimento sociale (Binbi, 1985).

Il tema del lavoro di cura diviene centrale nella riflessione sociologica a partire dagli anni 70, tanto da essere definito da Saraceno (2009) il dilemma inespresso, emblema di una vera e propria contesa per la rivendicazione di diritti sociali, terreno di conquista fatto di ambivalenze e paradossi. Questo tema, oggi, rappresenta uno snodo fondamentale nei dibattiti politici ed accademici, dal momento che il care, la cura non ha connotazioni nazionali e si configura come un nuovo bisogno sociale espresso a tutte le latitudini, in equilibrio tra partecipazione al mondo del lavoro e doppia presenza, tra responsabilità pubbliche e private. Un equilibrio ancora poco stabile visto che la conciliazione rimane ancora un problema al femminile e che l'affermazione di uno modello di famiglia dual earner è ancora un work in progress, una maratona senza traguardo, soprattutto nei Paesi mediterranei.

A tal proposito, sembra utile ricordare che le performance occupazionali delle donne seguono perfettamente l'usuale distinzione dei regimi di welfare state (Esping Andersen, 1990; Ferrera, 1993; Moreno e Salido, 2007), con le note differenze tra paesi nordici e meridionali. Pertanto, nonostante gli innegabili progressi raggiunti dalle donne nel mercato del lavoro, la 
conciliazione tra lavoro e cura appare una questione ancora irrisolta (Del Boca, 2009), nella misura in cui il modello male bredwinner ha lasciato il posto ad un modello in cui spicca la figura di un 'percettore e mezzo' o un 'percettore di reddito a tre quarti' (Saraceno, 2009), Iontano da quello che doveva essere il modello paritario, dual earner, a doppio reddito. Come hanno dimostrato alcuni studi comparati di settore (Raitano, 2009), si ha l'impressione che, nonostante i numerosi passi in avanti compiuti in direzione della parità di genere, le donne rimangano dei 'breadwinner intermittenti' (Glenn, 2002) e che, anche in famiglie a doppio reddito, siano le principali responsabili dei lavori domestici e della cura dei membri non autosufficienti. Anche per questo, il tema della conciliazione tra responsabilità familiari e lavoro remunerato è ancora oggi la parola d'ordine nelle agende politiche internazionali (Saraceno e Naldini, 2011), questione chiave della parità tra uomini e donne (Bernardi e Caldarini, 2009).

Tutto questo ha favorito la nascita e il sedimentarsi di una cultura defamilizzatrice per le donne, soprattutto nell'ambito domestico e di cura dei bambini, nella misura in cui il proliferare di strutture per l'infanzia ha consentito a molte madri di lavorare. Non è strano, quindi, soffermarsi sulla conciliazione quando si parla di cura e responsabilità familiari: essa entra inevitabilmente in gioco, (Saraceno, Naldini, 2011:8). Anche oggi, infatti, le capacità e le pratiche intese a raggiungere un equilibrio tra lavoro e vita familiare sono intimamente connesse con le aspettative riguardo a chi deve sottrarre tempo al lavoro per dedicarsi alla cura (Hobson, Dunvander, Halldén, 2009).

In letteratura vi è unanime accordo nel ritenere che la domanda di assistenza aumenterà in modo esponenziale nei prossimi decenni e l'altra parte del welfare, Stato e mercato, sarà certamente chiamato in causa, visto che la fonte tradizionale di cura, costituita da membri della famiglia, rischia di prosciugarsi, facendo diventare il tema della cura legata all'invecchiamento il divide et impera tra mondo del lavoro e gestione della cura, tra ruoli e aspettative di vita legate ai caregiver (Sarasa, 2007 b). D'altra parte, l'aumento delle richieste di cura fa avanzare l'ipotesi che i modelli di salute in vecchiaia, in previsione di una Long Term Care, richiederanno coadiutori appositamente formati (Rodrigues e Schmidt, 2010), dando rilevanza sociale a quella fino ad ora è sempre stata una questione femminile (Moreno, Salido, 2007).

Emerge in questo modo la necessità di una riconfigurazione degli schemi concettuali e interpretativi della cura, non più sovrapponibili alle tradizionali questioni sulle differenze di 
genere: sebbene i progressi nella direzione dell'uguaglianza siano evidenti, parallelamente si affermano nuove forme di esclusione non sempre coincidenti con queste ataviche differenze ma che con queste si fondono, si rigenerano e si moltiplicano (Sabbatini, 2009).

\section{Welfare transnazionale tra bisogni e responsabilità di cura}

Nel dibattito maturato attorno al concetto di cura, alla redistribuzione e alla ripartizione delle responsabilità tra pubblico e privato e tra uomini e donne, emergono visioni differenti circa la tematizzazione della parità di genere (Borchorst, Siim, 2009). Ogni tentativo di revisione finisce però con il riprodurre uno schema interpretativo dualistico che contrappone maschile e femminile, vita privata e pubblica, mercato, Stato e famiglia. Ogni proposta contiene in sé i semi di una ri/naturalizzazione delle differenze che finisce per limitare sempre più lo spazio di autonomia dei singoli, dando all'intervento dello Stato un carattere dirimente (Bimbi 2009). Le sfide costantemente sferrate e consumate a livello internazionale rendono ancora più incerta la definizione di benessere e dubbie le garanzie per $\mathrm{i}$ beneficiari; in realtà è sempre più difficile stabilire chi ne abbia diritto e a quali gruppi sociali possa essere esteso e soprattutto come esso possa essere salvaguardato in politiche e forme di cooperazione nazionali e transnazionali (Borchorst, Siim, 2009).

Nei dibattiti contemporanei poi il concetto di cura è stato rapportato anche ad altre questioni come l'etnicità e la globalizzazione, dimostrando come le disuguaglianze nella distribuzione della cura divengano globali in società complesse e multiformi. La domanda di cura proveniente dai paesi economicamente avanzati ha dato vita ad un processo di divisione internazionale del lavoro, in cui la compensazione dell'ormai acclarato 'deficit di cura' dei paesi sviluppati si risolve spesso attraverso l'importazione del lavoro d'amore e di cura dai paesi poveri a quelli ricchi (Eherenreich, Hochschild, 2002). In questo contesto, la divisione internazionale del lavoro riproduttivo diventa un tema importante per comprendere il mutamento dei processi migratori in Europa, per alcuni versi funzionali al lavoro di cura e coerenti con gli obiettivi di governance (sia dei paesi esportatori che di quelli importatori) e di una certa rilevanza anche rispetto al welfare. I presupposti e le dinamiche dei processi migratori connessi al lavoro domestico diventano una delle alternative possibili, una delle opzioni nel sistema dei servizi alla persona, soprattutto laddove il welfare è maggiormente fragile e frammentario (come succede in alcuni Paesi dell'area Mediterranea: Grecia, Spagna e Italia), caratterizzato da una persistente carenza di servizi pubblici e da uno scarso coordinamento tra i tradizionali fornitori di welfare (Ascoli, Ranci, 2003). In questo modo nei 
contesti in cui vi è una specifica richiesta di supporto privato alla cura, il sistema di catene migratorie (Hochschild, 2000 b) finisce per essere un potente strumento di conciliazione per le donne occidentali, capace di sostenere la doppia presenza, nella misura in cui il lavoro di donne migranti favorisce la realizzazione personale e la partecipazione al mondo del lavoro delle donne europee. II lavoro familiare finisce per diventare una nicchia occupazionale per i lavoratori migranti, fortemente legittimato nel senso comune come un lavoro tradizionalmente strutturato su asimmetrie e differenze e sempre più richiesto per ragioni ben note: bassa qualificazione, scarsa disponibilità della popolazione autoctona e per certi versi parziale convenienza dal punto di vista fiscale.

Si delineano dunque, nuove forme di stratificazione del mercato del lavoro, connesse all'immigrazione, nel mentre si creano nuovi equilibri e nuove dinamiche, che denotano cambiamenti cultutali e ulteriori processi di differenziazione nella sfera privata degli individui e delle famiglie. Anche gli ambiti privati divengono spazi globalizzati, crocevia di vecchie e nuove forme di interiorizzazione e riproduzione di disparità in cui l'astrazione e I'indeterminatezza della dimensione globale si respira e si materializza nelle dimensioni micro e intime del quotidiano (Barazzetti, 2007). Sicuramente, i profili di rischio emersi nelle società contemporanee hanno reso sempre più problematico 'per le famiglie' il fatto di occuparsi autonomamente dei bisogni di cura, finendo per indebolire la loro tradizionale capacità di self servicing, portandole sempre più verso soluzioni esterne e all'acquisto di servizi sul mercato (Ascoli e Ranci, 2003). Tutto ciò ha comportato un ripensamento delle responsabilità collettiva e pubbliche, in conseguenza dei grandi cambiamenti socio-demografici cui si è andata aggiungendo la preoccupazione per la carenza di manodopera femminile non retribuita (Leira, 2002).

L'assunzione di responsabilità pubblica su questi temi ha assunto connotazioni abbastanza diverse da un paese all'altro (Lewis, 1993; Orloff, 1993). In altre parole, i diversi modelli di welfare state hanno tracciato confini assai differenti tra pubblico e privato e, in relazione al proprio sistema di ideologie, norme e valori, hanno rafforzato e dato stabilità all'una o all'altra sfera, finendo per legittimare le relazioni di potere tra i generi implicitamente presenti in ciascuna di esse. Daly e Lewis (1998) ritengono, infatti, che i regimi di welfare abbiano stipulato il contratto di genere seguendo strade diverse e si siano strutturati a seconda del grado di familismo/defamilizzazione (Leitner, 2003; Saraceno e Keck, 2008) che hanno perseguito o supportato, nonché in base alla combinazione tra questi approcci. 
Sicuramente la defamilizzazione appare una soluzione vincente nella misura in cui supporta la famiglia e in particolare le donne nel lavoro domestico e di cura, facilitando il loro l'accesso al mercato del lavoro e ad una posizione lavorativa stabile (Esping Andersen, 1999; Gornick, 2001) nel mentre il familismo sostenuto, una sorta di porta secondaria della defamiliarizzazione, affianca la famiglia attraverso un pacchetto di politiche ad hoc (trasferimenti in denaro, congedi genitoriali, indennità di maternità).

L'assenza di servizi, la mancanza o la debolezza delle politiche sociali friendly family finisce per produrre quello che Saraceno e Keck (2008) definiscono familismo di default. Esso rappresenta uno snodo cruciale che, a seconda della strutturazione del mercato dei servizi e della cultura familiare, può portare ad un completo assorbimento e mantenimento delle responsabilità di cura nella famiglia e nella parentela o imboccare la strada della defamilizzazione tramite mercato. Quest'ultimo aspetto è molto importante perché ha a che fare con il difficile bilanciamento tra il sovraccarico connesso con forme di assistenza a lungo termine e i costi, spesso onerosi, legati alla professionalizzazione delle attività di cura e alla necessità di ricorrere a persone adeguatamente formate nel campo assistenziale (Torns, 2005).

Si apre, così, uno spaccato molto interessante che da un lato ha a che vedere con le solidarietà intergenerazionali e dall'altro con le differenze di genere e con un sistema di distribuzione delle responsabilità tra generazioni di donne (Murillo, 1995). Tali reti si organizzano, secondo criteri diversi a seconda dei modelli familiari, attraverso strategie tese alla salvaguardia dei propri confini anche quando essi appaiono sempre più trasversali (Hochschild, 2003). Nel ricorso al mercato, generalmente, ci si affida all'ausilio di altre donne, coordinando e organizzando le attività e i rapporti con i servizi pubblici, mantenendo un alto grado di coinvolgimento e svolgendo un'azione regolativa e di coordinamento (Caixeta et al., 2004; Suarez, 2004).

La ricerca sul welfare, sia da una prospettiva femminista che comparativista, ha inoltre ampiamente documentato come nei paesi in cui la rete dei servizi pubblici a favore di soggetti dipendenti (bambini e anziani) è ben articolata (come nel caso dei Paesi scandinavi e in parte della Francia e del Belgio) aumenti fisiologicamente la percentuale di madri lavoratrici (Anttonen, Sipilå, 1996) e analogamente diminuisca la quota di anziani che vivono con i figli (Esping Andersen, 1999). Queste esperienze rappresentano l'eccellenza ma anche 
un'eccezione nel panorama europeo che conferma la regola di una impostazione gender based delle politiche di welfare soprattutto dei paesi dell'area mediterranea (Anttonen, Sipilå, 1996).

In linea generale, è evidente come il grado di sviluppo delle politiche sociali nei diversi Paesi si possa misurare non solo dal livello di interesse delle autorità pubbliche nel rispondere ai bisogni di cura ma anche dalla quota di responsabilità attribuita alle famiglie (Ranci, 2003). In realtà con la complessificazione del vivere sociale gli interrogativi aumentano e sembra sempre più difficile trovare la giusta connessione tra uguaglianza e diritto di scelta, universalismo e selettività, responsabilità collettive ed individuali. Anche da un punto di vista teorico, occorre misurarsi con il fatto che la molteplicità di condizioni e modi di vivere sia ormai una caratteristica istituzionalizzata che necessità di una funzione di raccordo. In questa prospettiva, l'esternalizzazione del lavoro di cura acquista centralità in quanto consente di tessere e ricomporre il tessuto delle tutele frammentato dalla crisi, si pone in continuità con le tradizionali caratteristiche del lavoro domestico e consente lo svilupparsi di quella funzione di raccordo sempre più rilevante nelle società contemporanee, liberando energie fisiche e mentali tese alla salvaguardia dei legami affettivi tra le generazioni (Balbo, 1978).

\section{Referencias bibliográficas}

ABODERIN, I. (2005): 'Conditionality' And 'Limits' Of Filial Obligation, Working Paper Number WP205 pp.1-27, Conceptual levers for developing a better understanding of the motivational basis and societal shifts or patterns in old age family support, Oxford, Oxford Institute of Again.

ANTTONEN A., SIPILÄ, J. (1996): European Social Care Services: Is It Possible to Identify Models?, Journal of European Social Policy n. 6, pp. 87-100.

ASCOLI, U., RANCI, C. (2003): // Welfare mix in Europa, Roma Carocci.

BALBO, L. (1978): La doppia presenza, Inchiesta n.32, pp. 3-11.

BARAZZETTI, D. (2007): C'è posto per me? Lavoro e cura nella società del non lavoro, Milano, Guerini e Associati.

BERNARDI, N., CALDARINI, C. (2009): Le pari opportunità nelle politiche sociali dell'Ue. Tappe principali e analisi comparata dei regimi di genere di nove paesi, Italian Journal of Social Policy n.2, pp.217-249. 
BIMBI, F. (1985): La doppia presenza: diffusione di un modello e trasformazione dell'identità, in Balbo L., Bianchi M., Ricomposizioni. Il lavoro di servizio nella società della crisi, Milano, Franco Angeli.

BIMBI, F. (2009): Genere - Donna/Donne. Un approccio eurocentrico e transculturale, parola chiave in Italian Journal of Social Policy, vol. 2, pp.251-296.

BORCHORST, A., SIIM, B. (2009): Uno Sguardo di genere sul concetto di welfare, Italian Journal of Social Policy vol.2, pp. 19-44.

BRANDT, M. (2011): Intergenerational Help and Public Assistance in Europe. A Case of Specializzation, Mea (Mannheim research Institute for the Economics of Again), 236:2011, pdf downloaded from http://share-project.org.

CAIXETA, L., GUTIÉRREZ, E., TATE, S., VEGA, C. (2004): Hogares, cuidados y fronteras...derechos de las mujeres inmigrantes y conciliación, Madrid, Traficantes de Sueños.

CAMPILLO POZA, I. (2010): Políticas de conciliación de la vida laboral y familia en los regímenes de bienestar mediterráneos: los casos Italia y España, Política y Sociedad n. 47/1, pp. 189-213.

CASTLES, F. (1995): Welfare State Development in Southern Europe, West European Politics n. 28/2, pp.291-313.

CIARINI, A. (2011): Alla ricerca di nuove equilibri. Lavoro di cura, conciliazione e intervento pubblico nei sistemi di welfare europei, Italian Journal of Social Policy n.2, pp.257278.

CLIMENT, S. (2008): Gender and the migration and the legal frame of defamilialization in the Spanish welfare state, working paper n. 5.2, Denmark, CINEFOGO.

DALY, M., LEWIS, J. (1998): Introduction. Conceptualising Social Care in the Context of Welfare State Restructuring pp. 1-24, in Lewis J. (eds), Gender, Social Care and Welfare States, Aldersgot/London, Ashgate.

DALY, M., LEWIS, J. (2000): The concept of Social Care and the Analysis of Contemporary Welfare States, British Journal of Sociology n. 51, pp. 291-298.

DEL BOCA, D. (2009): Italia. partecipazione al lavoro, vincoli e strategie, Italian Journal of Social Policy n. 2, pp. 145-164.

DIEKMANN, A. (2004): The Power of Reciprocity. Fairness, reciprocity, and stakes in variants of the dictator game, Journal of Conflict Resolution n. 48/4, pp. 1-19. 
DOERINGER, P.B., PIORE, M.J. (1971) Internal Labor Markets and Manpower Analysis, London, Sharpe Publishers.

EHRENREICH B., HOCHSCHILD, A. (2002): Introduction, in Ehrenreich B., Hochschild A. (eds), Global woman. Nannies, maids and sex workers in the new economy, pp 1-13, New York, Henry Holt and Company.

ESPING ANDERSEN, G. (1990): The Three Worlds of Welfare Capitalism, London, Polity Press.

ESPING ANDERSEN, G. (1996): Welfare State in Transition, National Adaptions in Global Economies, London, Sage.

ESPING ANDERSEN, G. (1999): Social Foundations of Postindustrial Economies, Oxford, Oxford University Press.

EUROSTAT (2009): Reconciliation between Work, Private and Family Life in the European Union, in htpp//epp.eurostat.ec.europa.eu.

FERRERA, M. (1993): Modelli di solidarietà. Politica e riforme sociali nelle democrazie, Bologna, II Mulino.

FERRERA, M. (1998): Il modello sud-europeo di welfare state, Rivista di Scienza Politica n. 26/1, pp. 67-101.

FINCH, J., MASON, J. (1993): Negotiating family responsibilities, London, Routledge.

FLAGUER, L. (2000): Las políticas familiares en una perspectiva comparada, Barcelona, Fundación La Caixa (Colección Estudios Sociales).

FLAGUER, L. (2001): Family policy and welfare state in southern Europe, Barcelona, Institut de Ciencies Politiques i Socials.

GLENN, E. N. (2000): Creating a Caring Society, Contemporary Sociology n. 29/1, pp. 84-94.

GORNICK, J.C., MEYERS, M.K. (2001): Lesson-Drawing in Family Policy: Media Reports and Empirical Evidence about European Developments, Journal of Comparative Policy Analysis, Research and Practice n. 3, pp.31-57.

GOULDNER, A. W. (1960): The norm of reciprocity: a preliminary statement, American Sociololical Review n. 25, pp.161-78.

HABERMAS, J. (1998,): Lotta di riconoscimento nello stato democratico di diritto, pp. 63-100, in Taylor (eds), Multiculturalismo, lotte per il Riconoscimento, Milano, Feltrinelli.

HERNES, H. M. (1987): Welfare States and Women Power, Oslo, Norwegian University Press.

HOBSON, B., DUVANDER, A.Z., HALLDÉN, K. (2009): La conciliazione degli uomini e delle donne. Capacità e pratiche, Italian Journal of Social Policy n.2, pp. 79- 118. 
HOCHSCHILD, A. (2000 a): Global Care Chains and Emotional Surplus Value pp. 235-256, in Hutton W., Giddens A. (eds), On the Edge. Living with Global Capitalism, London, Jonathan Cape.

HOCHSCHILD, A. (2000 b): The Nanny Chain, The American Prospect n. 3, pp.32-36.

HOCHSCHILD, A., EHERENREICH, B. (2003): The Commercialization of Intimate Life. Nores from Home and Work, Berkeley, University of California Press.

KITTAY, E.F. (1999): Love's Labor: Essay on Women, Equality and Dependency, New York, Routledge.

LAND, H., ROSE, H. (1985): Compulsory Altruism for Some or an Altruistic Spciety for All? pp. 7496, in Bean P., Ferris J., Whynes D. (eds), In Defence of Welfare, London, Tavistock.

LEIBFRIED, S. (1992): Towards a European Welfare States? pp.245-279, in Ferge Z., Kalbery J. E. (eds), Social Policy in a Changing Europe, Boulder, Westview Press.

LEIRA, A. (1992): Welfare States and working mothers. The Scandinavian experience, Cambridge, Cambridge University Press.

LEIRA, A. (2002): Working parents and the welfare state, Cambridge, Cambridge University Press.

LEIRA, A., SARACENO, C. (2002): The multidimensional care concept, pp.55-83, in Hobson B., Lewis J., Siim B. (eds), Contested concepts in gender and social politics, Cheltenham, Edward Elgar.

LEITNER, S. (2003): Varieties of Familialism: The Caring Function of the Family in Comparative Perspective, European Societies n.5, pp. 353-375.

LEWIS, J. (1993): (eds), Gender and social policies in Europe, Aldershot, Edward Elgar.

LEWIS, J. (1998): Gender, social care and welfare state restructuring in Europe, London, Ashgate.

LEWIS, J. (2002): Gender and welfare state change, European Societies n.4, pp. 331-357.

MARTIN, C. (1996): Social Welfare and protection by the family in Southern Europe: Are Their Any Specificities? Paper presented at the Conference Comparing Social Welfare System in Southern Europe, Florence European University, 22-23-24 February.

MARTÍN PALOMO, M. T. (2008): Los cuidados y las mujeres en las familias, Politica y Sociedad, n. $45 / 2$, pp. 29-47. 
MORENO, L. (2001): Spagna, una via intermedia allo sviluppo del welfare, Argomenti $\mathrm{n}$. 3/2001, pp. 115-143.

MORENO, L. (2002): Bienestar mediterráneo y "supermujeres", Revista Española de Sociología n. 2 , pp. 41-57.

MORENO, L. (2004): Spain's transition to new welfare: a farewell to superwomen pp. 133-157, in Taylor Gooby P. (eds), New Risks, New Welfare: The Transformation of the European Welfare State, New York, Oxford University Press.

MORENO, L., SALIDO, O. (2007): Bienestar y políticas familiares en España, Política y Sociedad n. 44/2, pp. 101-114.

MORENO, L., SARASA, S. (1992): The Spanish "via media" to the development of the Welfare State, Madrid, IESA (CSIC), DT 92-13 pdf download from http://www.iesam.csic.es/doctrab.htm.

MURILLO, S. (1995): Espacio doméstico: el uso del tiempo, in Tobío L., Denche C. (eds), EI espacio según el género, ¿̇un uso diferencial?, Madrid, DGM.

NALDINI, M. (2002): Le Politiche Sociali e la famiglia nei Paesi Mediterranei. Prospettive di analisi comparata, Rivista Stato e Mercato n. 64, pp.73-99.

NALDINI, M. (2006): Le Politiche Sociali in Europa. Trasformazioni dei bisogni e risposte di policy, Roma, Carocci.

O'CONNOR (1993): Gender, Class and Citenzenship in the Comparative Analysis of Welfare State Regimes: Theoretical and Methodological Issue, British Journal of Sociology n. 44/3, pp. 501-518.

ORLOFF, A. S. (1993): Gender and Social Rights of Citizenship: The Comparative Analisis of Gender Relations and Welfare State, American Sociological Review n. 58/3, pp. 303329.

PAPERMAN, P. (2005) Les gens vulnérables n'ont rien d'exceptionnel pp. 281-297, in Paperman P. Laugier D., Le souci des autres, éthique et politique du care, Paris, EHESS, Raisons Practiques.

PIPERNO, F., Tognetti Bordogna M. (eds) (2012): Welfare transnazionale: la frontiera esterna delle politiche sociali, Roma, EDIESSE.

RAITANO, M. (2009): Differenze di genere nel mercato del lavoro dei paesi della Ue-15, Italian Journal of Social Policy n. 2, pp.165-187. 
RANCI, C. (2003): L'economia mista dei servizi di cura in Europa, pp. 45-65, in Ascoli U., Ranci C. (eds), Il welfare mix in Europa, Roma, Carocci.

RODRIGUES, R., SCHMIDT, A. (2010): Paying for Long-term Care, Mea (Mannheim research Institute for the Economics of Again), pdf downloaded from http://share-project.org.

SABBATINI, A. (2009) Donne e welfare. Una cittadinanza incompiuta. Presentazione del libro, Italian Journal of Social Policy n.2, pp. 7-15.

SAINSBURY, D. (1994): (eds) Gendering Welfare States, London, Sage Publications.

SAINSBURY, D. (1996): Gender Equality and Welfare State, Cambridge, Cambridge University Press.

SAINSBURY, D. (1999): Gender and Welfare StatesRegimes, Oxford, Oxford University Press.

SARACENO, C. (1994): The ambivalent Familism of the Italian Welfare State, Social Politics n. 1, pp. 60-82.

SARACENO, C. (2009): Genere e cura: vecchie soluzioni per nuovi scenari?, Italian Journal of Social Policy n. 2., pp.53-75.

SARACENO, C. (2010): Social inequalities in facing old-age dependency: a bi-generational perspective, Journal of European Social Policy n. 20/1, pp. 32-44.

SARACENO, C., KECK, W. (2008): The Institutional Framework of Intergenerational Family Obligations in Europe: A Conceptual and Methodological Overview, pdf downloaded from http://www.multilinks-project.eu/info/papers.

SARACENO, C., NALDINI, M. (2011): Conciliare famiglia e lavoro. Vecchi e nuovi patti tra sesso e generazioni, Bologna, II Mulino.

SARASA, S. (2007): The women's conciliation of adult care and employment in different welfare regimes, in Esping Andersen G., (a cura di), Family Formation and Family dilemmas in contemporary Europe, pp.1-33, Bilbao, Fundación BBVA.

SIIM, B. (2000) Gender and Citizenship. Politics and Agency in France, Britain and Denmark, Cambridge, Cambridge Uuniversity Press.

SUÁREZ, L. (2004): Transformaciones de género en el campo transnacional. El caso de mujeres inmigrantes en España, La Ventana n. 20, pp. 293-331. 
THOMAS, C. (1993): De-constructing Concepts of Care, Sociology n.27/4, pp. 649-669.

TORNS, T. (2005): De la imposible conciliación a los permanentes malos arreglos, Cuadernos de Relaciones Laborales n. 23/1, pp.15-33.

VAN KERSBERGEN, K. (1995): Social Capitalism. A Study of Christian Democracy and the Welfare State, London-New York, Routledge.

WAERNESS, K. (1984) Caring as Women's Work in the Welfare State, in Holter, H. (eds), Patriarchy in a Welfare Society, Oslo, Universitetsforlaget.

WÆRNESS, K. (1987) On the Rationality of Caryng, in Sasson A.S. (a cura di), Women and the State, Londra, Hutchinson, pp. 207-234.

WAERNESS, K. (1996): On The Rationality of Caring, in Showstack Sasson A. ( a cura di), Women and State. The Shifting Boundaries of Public and Privare, Londra, Hutchinson, pp. 20723 